\title{
Research on the System Structure of University Digital Campus Jianping Zhang ${ }^{1, a,}$ Jie Zhou ${ }^{1, b,{ }^{*}}$ Qiumin Luo ${ }^{1, c}$ and Guangbin Fan ${ }^{1, d}$ \\ ${ }^{1}$ Dalian Jiaotong University, Dalian, China \\ azhangjp@djtu.edu.cn, bzj@djtu.edu.cn, ${ }^{c}$ lqm@djtu.edu.cn, dfgb@djtu.edu.cn
}

Keywords: Digital campus; System structure; Application system; Data resources

\begin{abstract}
With the rapid development of computer technology, network technology and communication technology and its extensive application in the field of education, the information construction of universities with the digital campus as a landmark project has been developing rapidly. Through the integration of information resources such as teaching, scientific research, management and life service, the digital campus provides all-round and personalized information service for teachers and students. It has become an indispensable infrastructure to comprehensively improve the quality of school running and the efficiency of running schools. Starting from the connotation of digital campus and the problems existing in the construction, this article analyzes the guiding ideology and principles of its construction, and constructs the system structure of digital campus. It has certain guiding significance for the practice of university digital campus construction.
\end{abstract}

\section{The Connotation of Digital Campus}

Digital campus is based on campus network, adopts unified standard and standard, integrates all kinds of resources in Colleges and universities, establishes a unified application service system through secure identity authentication.

It can be realized in the environment (including equipment, office space, study space, teaching space), resources (such as books, lectures and teachers and professional database courseware, global online professional information), activities (including teaching and learning, scientific research, management, service, office etc.) all digital and high information sharing. It can improve the efficiency of teaching, management and service, and ultimately establish a digital education environment with resources, services and security. Its main content can be explained as follows:

(1) The popularization and use of computers and the foundation construction of campus network are the premise and foundation of digital campus.

(2) Digitalization includes three levels: digitization of environment, digitalization of resources and digitalization of management.

(3) Digitalization will infiltrate into the teaching, scientific research, management and life service, and its core is around the training of talents

(4) Its ultimate goal and significance lies in the construction of higher education model suitable for the requirements of the information society.

\section{The Current Problems in the Construction of Digital Campus}

The construction of digital campus as a complex system and social engineering, there are a lot of problems at the technical level, capital level, cognition level, coordination level and personnel security level for ordinary universities. Mainly in the following aspects:

Information Standards are not Unified. Lack of scientific and unified information resources planning has not formed a unified information standard system. The same data is likely to be stored in multiple systems, and lack of authoritative data sources. Due to the absence of uniform data standards, real-time data exchange is difficult. 
Overflow of Application System. Each application department independently purchases the application system according to its own demand. Because of the lack of overall planning and technical differences lead to convergence difficulties. Users need to log on different systems according to different requirements, lack of unified entrance and one-stop platform, and the entire digital campus architecture is not perfect.

The Utilization Rate of Application System is Low. Many departments have established the application system, but due to the personnel information literacy problems can not be well used, facing "road without car", "car without goods" problem.

The Focus of Construction is still Concentrated in Infrastructure. The importance of integration of data and application systems to university management has not yet been recognized. The construction of digital campus is still at the low end of the road, and the stage of network hardware.

The Importance of the Information Departments is not Enough. At present, most of the information departments in universities mainly solve technical problems, however, it is not enough to carry out the popularization and application of digital campus in the whole school, and the implementation is not enough. Even some universities believe that the information departments are not be needful, the construction of information organization can not be carried out from the strategic development. [3]

\section{The Guiding Ideology and Principles of Digital Campus Construction}

At present, most universities in China are in the construction of digital campus, but the construction can not be blindly carried out, we must follow certain rules and guiding ideology. That should make full use of the existing hardware infrastructure, act serve as the fundamental purpose and let improve the level of university information as the ultimate goal. In the process of construction, we should fully consider the performance, maintenance, management, expansion, so that the application system has high scalability, and in a certain period of time can be sustainable development.

Unified Planning and Distribution Implementation. The construction of digital campus is a complex and systematic project, which requires a variety of technologies to be used together. At the same time, it involves all departments of the university, and it has large investment and long construction period. Therefore, at the beginning of the construction, universities should make overall planning according to their own and long-term development goals. That can meet the overall needs of the school. At the same time, the construction of digital campus is not accomplished overnight, which need a longer period of time and the implementation of the distribution. On the one hand, it can reduce the project risk, improve the success rate of the project, at the same time, it can also establish buffer for the implementation of the project, leaving more thinking and trial run time, so as to ensure that the project can meet the expectations of the universities.

Resource Integration and Strengthening Application. Many application systems in universities are isolated from each other, and cannot communicate with each other and share data, which leads to the isolation of information islands. Many system functions coincide with each other, belonging to repeated construction, which not only wastes resources, but also reduces work efficiency. Therefore, digital campus construction wants to make the data to create greater value only by achieving data sharing and establishing data links. Digital campus construction is for teaching, scientific research, management and life service, once separated from this, the construction of digital campus meaningless. Therefore, the construction of digital campus must be dominated by application.

Advanced Technology, Safe and Stable. The construction of digital campus lasts for a long time, and it should also be advanced and mature in a certain period of time. The advanced technology and systematic engineering are adopted. At the same time, the system architecture, system structure, database applications can not be ignored. That will ensure the stability of the system without fault operation. 
Easy Maintenance and Easy Expansion. Platform construction is a complex and wide system. After the operation stage, the later maintenance work will also have a great workload. Therefore, in the early stage, the late maintenance should be considered. At the same time, it needs to expand the interface for information construction, and it has good scalability. The combination and smooth transition of new and old technology should be fully considered. [2]

\section{The System Structure of Digital Campus [4]}

Under certain information security system and data standard, the system structure of digital campus can be divided into seven modules. Fig 1 .

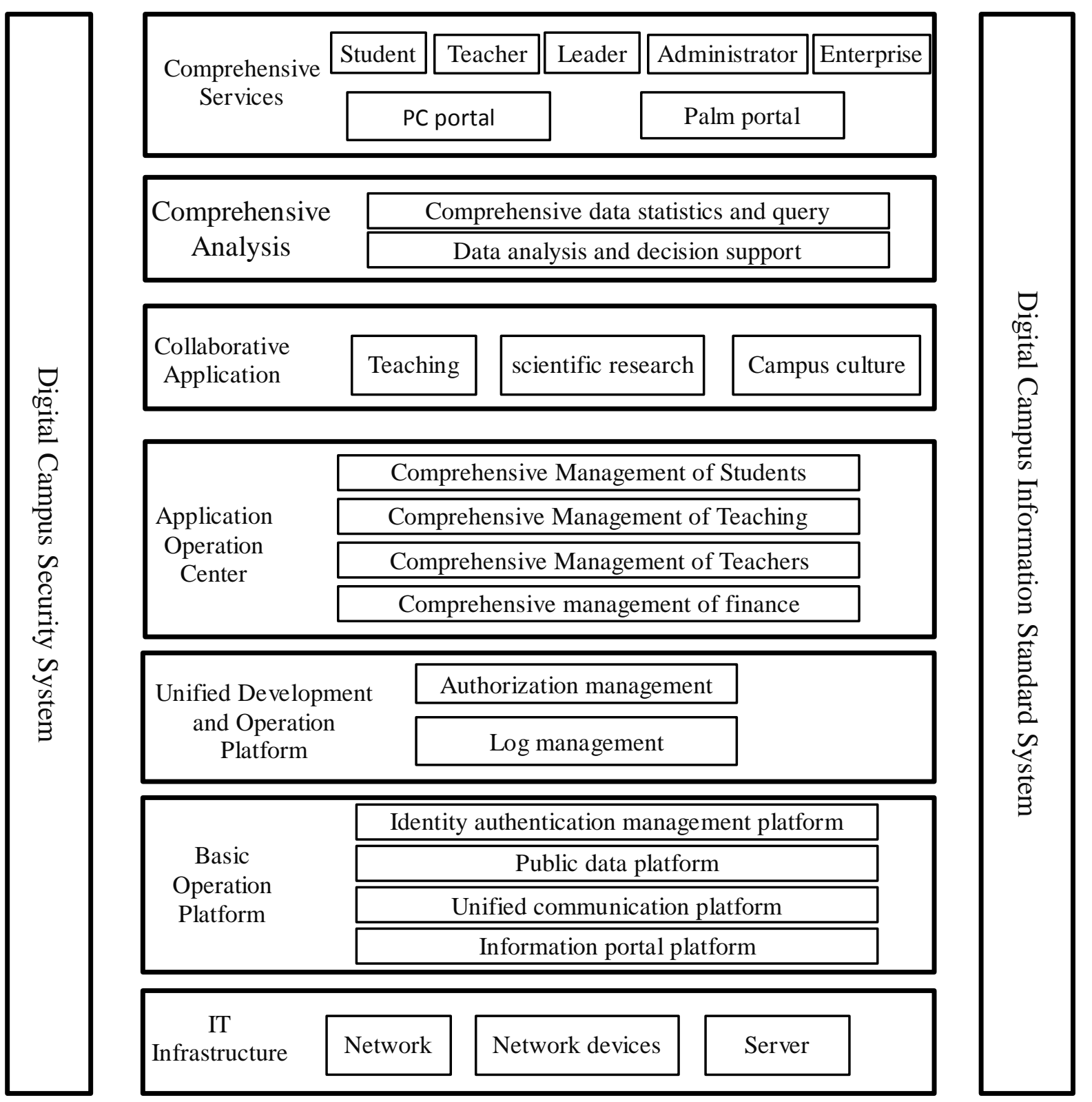

Figure 1. system structure of digital campus

IT Infrastructure. IT infrastructure construction is the hardware foundation of digital campus construction, involving wired, wireless networks, network equipment, servers and so on. Network devices include switches, routers and other devices. The server includes database server, Web server, backup server, mail server and so on. These hardware devices constitute the supporting environment of digital campus.[5] 
Basic Operation Platform. The basic operating platform is mainly software system. It mainly includes the following aspects: identity authentication management platform can unify storage of user authentication information and unify identity authentication. Public data platform can provide a warehouse for public data interaction. Unified communication platform can unify platform, direct communication mode, centralize and unify management platform communication. Information portal platform can unify information portal. [5]

Unified Development and Operation Platform. The platform needs a stable, reliable and safe operation platform to support, at the same time, business processes and management changes are everywhere. A flexible and fast development platform is required to ensure that applications are customized on demand. [5]

Application Operation Center. The comprehensive management mainly revolves around the teaching, the student management, the office and the human resources management. The digitized campus platform should be constructed which can integrate the campus information of each subsystem. Through the integration of the digital campus platform, it can integrate the information and process of the existing management system. A. Comprehensive Management of Students. Because of the large number of students and the rapid change of business requirements, the management of students often needs to coordinate multiple departments to complete a task, such as new orientation, school leaving, scholarship and so on. The management functions of the system need to be considered from the perspective of the school as a whole. It is not easy to manage independently in the department or in the student's category, otherwise, on the one hand, the school construction system can not get the desired effect, on the other hand, it will build system repeatedly. B. Comprehensive Management of Teaching. Teaching management provides a unified online work platform for educational administration departments, teaching departments, teachers, students and related functional departments. The main coverage of curriculum management, teacher information management which will dock with teachers information management, and personnel, financial and other systems, status management which will dock with the student management system, financial management system, the management of teaching plan which will dock with the quality course construction and teaching resource library system, course management which will dock with course scheduling system and the teachers information management, educational administration management, the daily examination management and performance management. [6,7] C. Comprehensive Management of Teachers. This system focus on the construction of teaching staff, It provides integrated management and one-stop service platform for teachers. D. Comprehensive management of finance. Financial comprehensive system includes financial information management, asset information management, equipment management, logistics management and so on.

Collaborative Application. Collaborative application services mainly focus on the two core business contents (teaching and research) in universities. Through collaborative application to improve the university's scientific research ability, service social ability, and teaching quality.

Comprehensive Analysis. The construction of various application systems in universities plays a positive and effective role in the development and promotion of school business. But the construction of teaching management system, students management system, person management system and other application systems only focus on their own application or their own business departments. A comprehensive inquiry system has not yet be established which covered the overall university. The leaders and managers can not understand the overall school situation. Application systems are even less likely to provide the basis for school decisions. Comprehensive analysis use data extraction tools and data mining tools such as data warehouse technology. It can comb the school situation data, accumulate information resources, standardize information management, strengthen the monitoring quality of teaching and promote the coordinated operation of various departments of the university to improve the school management level.

Comprehensive Services. Through the campus information portal, it can provide one-stop, personalized, comprehensive information service for the teachers and students, leaders, cooperative enterprises, alumni and other service objects. All the functions and applications provided are 
released through the comprehensive services system. The service content is designed for the service object, and is displayed on the platform of the school information portal.

Digital Campus Supporting Environment System. A. Digital Campus Security System. The establishment of a better security authentication mechanism is a good role in ensuring the construction of digital campus. It is support to ensure its reliable operation. Therefore, in the process of digital campus design, it is necessary to comprehensively plan the entire security service system, from hardware, software, personnel, systems, information, data to the other aspects to ensure the safety [8]. B. Digital Campus Information Standard System. Without unified information standard, it is difficult to integrate each subsystem. Therefore, the establishment of standardized work is one of the core tasks of digital campus. The standards mainly include the following aspects: information standards, coding standards (data standardization), management specifications, etc.

\section{Summary}

The construction of digital campus is complex system engineering. It can be influenced by the original environment of the school. Funding, policy and other aspects of support also restrict the progress of the work. At the same time, the technology also needs the information builders to continue to explore and perfect in practice. It needs continuous construction and integration to adapt the requirement of the development of the university.

\section{Reference}

[1] Jiang Li. The Plan and Construction Design of Digital Campus: The Case at the Caotang Campus of Xi'an University of Architecture and Technology[D]. Xi'an University of Architecture and Technology 2014. P8

[2] Yange Pang. Research on construction scheme and technology of Digital Campus: The Case at the Cangzhou Medical College[D]. TianJin Polytechnic University 2017.P16,19,

[3] Zhaohai Ran, Digital Campus Planning and Practice [D].Chang'an University 2013. P10

[4] Qiang Cao. Design and Implementation of Digital Campus[D]. Qingdao Technolgical University 2014 P33

[5] Shichao Zhang. Design and Realization of Digital Campus[D]. University of Electronic Science and Technology of China 2014 P33,34

[6]Ruihua Zhu. The Research of Designing and Developing on Educational Administration Information System in Vocational Institute[D]. Tianjin University 2010

[7]Liyan Ma. The Design of Educational Management System in Lianyungang Teachers' College[D]. East China Normal University 2009

[8] Xu Zhang, Xiaoxia Huang. Research and Implement of MIS-Based User Permission Management[J]. Modern Computer 2008.5 